\title{
Closing the Gap for Young Aboriginal and Torres Straight Islanders, Utilising Today'S Technology and Moving Forward Together
}

\author{
Klaus Martin Beckmann ${ }^{1, *}$, Adrian Hepi ${ }^{2}$ \\ ${ }^{1}$ School of Medicine, Logan Campus, Griffith University, Logan, QLD 4131, Australia \\ ${ }^{2}$ School of Public Health, Tropical Medicine and Rehabilitation Sciences, James Cook University, Australia \\ *Corresponding Author: martinbeckmannnn@hotmail.com
}

Copyright $(\underset{C}{2013}$ Horizon Research Publishing All rights reserved.

\begin{abstract}
Australia is talking about 'Closing the Gap'.This gap relates to differences in qualitative and quantitative criteria, comparing Aboriginal and Torres Strait Islanders with non-indigenous people. We link internet access with prevention of trauma for young Aboriginal and Torres Strait Islanders in Queensland Australia. Some areas around the world are lagging behind with information technology (IT) development, so it appears do some of the rural and remote areas in Queensland Australia. We look at the gap in internet access and how potentially good IT access may have a beneficial effect on how trauma is perpetuated trans-generationally. Human society is seeing unprecedented acceleration in the development of faster and wider ranging options for communication. We show that lack of IT access is potentially a barrier to accessing self-motivated education and changing perceptions as to what may be acceptable parenting. We draw parallels with powerful internet-assisted groupings of individuals that triggered change. Some of these initiatives have been successful, some appear popular and widely acceptable,others not. Immediate communication was quintessential for individuals coming together. Self-motivated addressing of the cycle of abuse via internet social networking sites in Australia's remotest areas is unlikely without good IT access. Of course there are many other factors contributing to the prevalence of child abuse in remote parts of Australia. Although we are hopeful, it remains to be seen what effect the internet will have on moving forward together when even the remotest parts are well connected.
\end{abstract}

Keywords Internet, Social Networking, Trauma, Abuse, Prevention, Children, Young People, Aboriginal And Torres Strait Islander, Queensland, Australia

\section{Introduction}

We place 'the Gap' in its wider context. We investigate how a gap in provision of internet access may influence victims getting together and standing up for their protection. We also look at some of the models for behaviour modification for perpetrators of child abuse. To our knowledge this is the first paper that examines the potential of IT access, i.e. social networking sites, change in behaviours and Aboriginal and Torres Strait Islanders. Searches on Google and Google scholar in early 2013 using the search terms "Internet, social networking, trauma, abuse, prevention, children, young people, Aboriginal and Torres Strait Islander, Queensland, Australia" did not yield relevant results.

The magnitude of child abuse is highlighted in statistics from the Australian Institute of Health and Welfare [1]. Reports for 2011 show that Aboriginal and Torres Strait Islander children and young people (from now on referred to as "children") are over-represented in child protection and out-of-home care services if compared to other Australian children. Between 1 July 2010 and 30 June 2011, of 1000 Aboriginal and Torres Strait Island children "34.6" had child protection records of substantiated harm, or risk of harm from abuse or neglect. For non Aboriginal and Torres Strait Island children this number was " 4.5 " in 1000 . Child abuse is far too prevalent and even more so especially in the remotest parts of Australia.

With view to prevention we select a number of models that may have an impact on behaviours. We look at the victims and also the perpetrators. We focus on the North of Queensland, Aboriginal and Torres Strait Islanders living in remote and rural communities.

We know from the work by for example Nicola Tesla, Guglielmo Marconi and Samuel Morse that introduction of telecommunication technologies can lead to significant social impact. We examine the relevance of IT on community discussion and action. We explore what may work for children who have been victims. We give examples from other parts of the world where IT has been ever so powerful in triggering changes to set patterns. We link IT 
with development of an internal locus of control. We hypothesise that if there is a change in the locus of control to an internal locus that, with widely accessible communication tools and associated educational opportunities, this can spread and lead to a change in behaviour, eventually we hope increasing the safety of children. We offer possible solutions to appropriate networking and therewith hopefully reduction of tragedies in families.

We appraise the limitations and we offer potential solutions. Whilst we aim to be as comprehensive as possible, for the purpose of this paper, there are several aspects like the role of mobile phone economy and microcredit etc. from other continents that we have not included.

Where possible we aim to communicate in a style that we hope is similar to how other health professionals establish rapport, have meaningful culturally appropriate discussions with community councils, men's and women's groups and youth groups. By using these tools of traditional dialogue we aim to be understood and be accepted by Aboriginal and
Torres Straight Islander communities.

\section{Material and Methods}

This is an article based on empirical investigation. Findings are reported in a narrative style quoting evidence where relevant. The article has its origins in a debate between the authors. Adrian one night walked along the dunes in North Queensland and beyond the dunes saw lights flashing on the horizon. He went to investigate why and found indigenous youth on the beach throwing and catching their mobile phones in the air. He asked for what reason and the reply was that they wish to send text messages and reception is higher up. We feel there is a keen will amongst youth to use IT to communicate and network but there are difficulties with access in remote areas. We wonder what potential those youth will have once they have access to good internet connections.

\section{Proportion of deaths by age group}

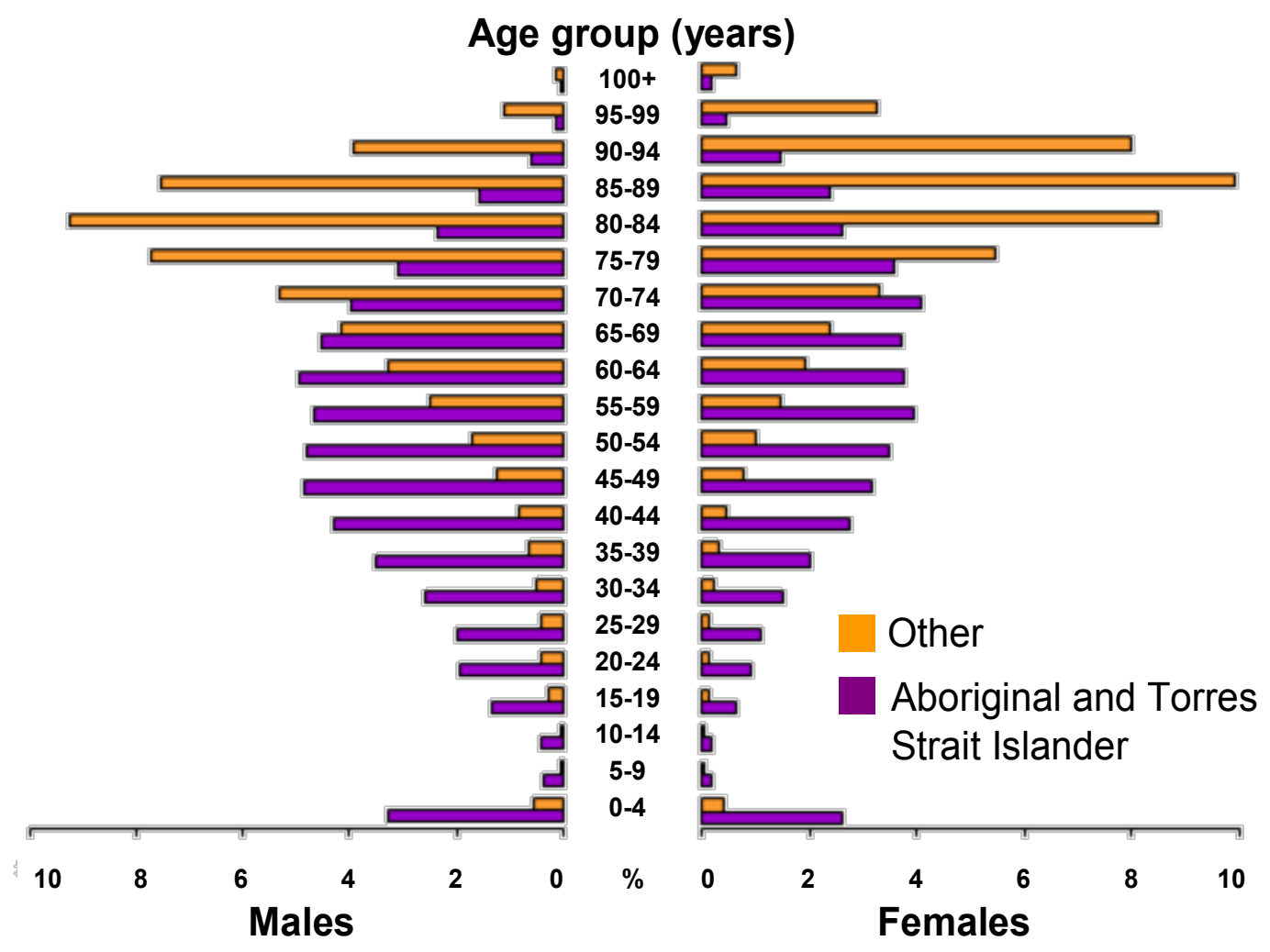

Proportion of Australian deaths (a) (b), Aboriginal and Torres Strait Islander status (c), Age group (d) and sex-2006-2008 Source: ABS 2006-2008 Deaths collection 


\section{Discussion}

\section{The Gap}

1.1 Closing the Gap is the endeavour of all Australia's states and territories. The gap relates to the major areas of life i.e. socio economic, health, education, social stratification and social mobility. Depending on the perspective Aboriginal and Torres Straight Islanders may be seen indeed as disadvantaged in today's society, however this will not apply to all domains. We can think of several examples where the gap is to the advantage of Aboriginal and Torres Strait Islanders.

1.2 Aboriginal and Torres Strait Islanders have a history of starting in Australia more than 40000 years ago. Consistent with Darwin's evolution theories [2] they have adapted to environments. Some of these environments can be extremely hostile to survival, at least at first glance to a newcomer. Today there are over 700 Aboriginal and Torres Strait Islander groups. In Queensland the Western and Eastern Islands consist of at least 16 different groups. These have their similar or separate stories, dreams, histories, identities and languages. Changes brought with new ways of living since the discovery of Australia by Europeans and immigration of additional ethnic groups, have without a doubt affected Aboriginal and Torres Strait Islanders. It is difficult to think of a domain where there has been introduction of something new to Australia where the minds and bodies of Aboriginal Torres Strait Islanders would not have adapted. Equally the later immigrants in the $18^{\text {th }}$ century have adapted to their new environments. An intriguing example for the difficulties for e.g. Caucasian immigrants adjusting and surviving in remote Australia can be seen in the Roeg's screen play 'Walkabout' [3]: orphaned Caucasian youths abandoned in remote Australia struggle with survival whilst an Aboriginal Youth makes excellent use of the resources in what may appear a hostile environment. Moreover Australian news regularly report Caucasians dying in the outback when, for example, stranded in a car but then not surviving, whilst there are no such regular reports of indigenous people dying in such situations. While the new immigrants may struggle to adapt to lifestyles that Aboriginal and Torres Strait Islanders have lead for thousands of years, the overall effects on morbidity and mortality are not as profound and hazardous as for Aboriginal and Torres Strait Islanders in their adaptation of Caucasian metropolitan lifestyles. We find that the gap is 2 sided, on the one side the gap is about immigrants sometimes lethal lack of survival skills in face of hardship and dangers in remote Australia and on the other side it is about the disadvantages of Aboriginal and Torres Strait Islanders in "Western Society". We now focus on the latter:

1.3 Bridging the gap is part of Australian government political targets. We refer to the department of Aboriginal and Torres Straight Islander Multicultural Affairs and Aboriginal and Torres Straight Islander Affairs document 'Closing the Gap' [4]. : reduce the gap in life expectancy within a generation, halve the gap in mortality for Queensland Aboriginal and Torres Straight Islander children under 5 within a decade, halve the gap in employment outcomes between Aboriginal and Torres Straight Islanders and non-indigenous Queenslanders within a decade, halve the gap in reading, writing and numeracy achievements for Queensland Aboriginal and Torres Straight Islander children within a decade, ensure all Queensland indigenous 4 year olds in remote communities have access to early childhood education within five years, and halve the gap for Queensland Aboriginal and Torres Straight Islander students in year 12 or equivalent attainment rates by 2020 .

1.4 Beyond such government targets the gap is monumental and broad. A good portrait of those differences between town and country is made explicit in Thornton screen play 'Samson and Delilah' [5]. This movie highlights differences in living circumstances, behaviours and access to mainstream western education, access to telephone and internet, differences in socioeconomic factors such as housing, employment, career opportunities, and access to networking in general, supervision, childcare, transport and nutrition.

1.5 We reference medicine where there are evidence-based epidemiological data from the Australian Bureau of Statistics which highlight the gap with respect to mortality [box 1]. This shows the discrepancy in life expectancy comparing Aboriginal and Torres Strait Islanders and non Aboriginal and Torres Strait Islanders. Recent examples of important areas in health where the gap is clearly demonstrated are: Graham [6] and Bygott [7] show evidence for the gap in sexually transmitted infection i.e. increased prevalence of Chlamydia and Gonorrhoea and Trichomonas in indigenous populations. Also mental health is affected: psychosis is more prevalent in remote indigenous areas of Queensland [8]. The Australian Heart Association provides evidence of increased morbidity [9].

\section{Prevention of Abuse - the Victims}

2.1.1. Preventative medicine differentiates between primary, secondary, tertiary and quaternary prevention. Ideally we wish that sequelae after severe adversity will not occur; hence a first goal is to stop misfortune before disaster actually happens. In this paper we focus on primary prevention: avoiding the onset of adverse effects right from the start by preventing what triggers the adverse effect. We believe primary prevention will have the greatest impact for potential victims because if not traumatised there will be no sequelae of trauma.

2.1.2. Failing primary prevention, vulnerability and resilience factors need to be considered. Whilst some children have resilience in the face of adversity and are less likely to suffer impairment following trauma, other children and young people will have vulnerability factors that can leave victims very impaired and suffering.

2.1.3. Bradford - Canon [10] described the 2 main trauma responses: internalising, associated with freeze response, and 
externalising behaviours, associated with fight flight response. The time to recovery is variable. It is not infrequent that recovery processes can last decades particularly if there was chronic and complex trauma with victims becoming increasingly aware and eventually seeking help in their thirties or forties. In Queensland it looks as if there may be a decrease in prevalence of abuse and consequences of abuse going from north to south, from remote areas to metropolitan areas. Children in remote Queensland are at risk of greater morbidity compared to their peers in southern Queensland. This is evidenced by aforementioned statistics from mental health, infectious diseases, physical health, sexually transmitted diseases, and likely applies also to abuse, and also areas like education and vocational activities.

2.1.4. Morbidity factors will affect short, medium and long-term bio- psychosocial-demographic outcomes and future opportunities. Philip et al [11] show evidence for impact of trans-generational abuse. Hence yesterday's victims may become today's abusers and today's victims may become tomorrow's abusers. Preventing and addressing trauma today is likely to change future generations' parenting styles. Appropriate parenting is likely to also positively affect individual's contribution to society, employment, education, identity, religion and culture. Such improvements are likely to positively influence social mobility and social status, opportunities, social class and costs to society as a whole.

2.1.5. Internal and external locus of control is a concept that goes back to Julian B. Rotter [12]. Typically with internal locus of control an individual will feel in charge of their life and destiny, with an external locus of control this feeling in charge is not so much with the self, but typically and often with something or someone else other than the self. Children often have an external locus of control. Toddlers and younger children go through a stage when they attribute blame externally [13]. Moreover typically children who are traumatised have learned to be a victim; hence such children are even more likely to have an ongoing external locus of control [14] Trauma also leaves victims with low self esteem, lack of trust and inertia especially if severely traumatised [15]. That can then lead to not speaking up when they reach the developmental age where values imposed by authority would typically be questioned. Furthermore an individual growing up, who has a history of feeling insecure, is likely to develop insecure or ambivalent disorganised attachment. This can present with erratic behaviour and thought patterns. This is likely to contribute to inadequate or delayed appropriate response to injustice [16]. Intervention can be difficult. Maybe good access to IT can help young people through learning, networking, and access to therapies. Online therapies are already well established when it comes to for example treatments of depression [17]. Avatar or similar programs may make it easier to engage children.

2.1.6. Children with illiteracy may prefer sites like You Tube which rely on audio and film. We suggest that once the national broadband network NBNCO [18] provides access to every community in Australia, be it via fibre, satellite or mobile network, this will also affect opportunities available in northern Queensland: it may be that IT will be taken up by at least some members of the community. This may then influence pre-existing values and perhaps lead to lobbying by and for the youngest members of indigenous societies. We believe that with the appropriate promotion closing the digital gap will lead to changes in parenting patterns, and all going well enhanced child protection in the short, medium and longer term. Eventually this can lead to a decrease in child abuse and in the long term to a decrease in trans-generational abuse.

\section{Prevention of abuse - the abusers}

3.2.1. Some behaviour is engrained, whether positive or negative. Child abuse is a negative behaviour. We suggest that, where so highly prevalent, child abuse may be at least in part a form of learned behaviour. Learned behaviour can be unlearned. There may be opportunities for behaviour modification. Child abuse is associated with psychosocial factors like unemployment, drug and alcohol use, education parameters and socio-demographic poverty matrices.

3.2.2. Substance abuse is known to be prevalent in remote Australia, unemployment is rife, access to statutory and non-statutory services are limited. Day to day activities generally are different compared to centuries ago. Some Aboriginal and Torres Strait Islander individuals may have lost sense of identity and connectedness to their people and past. In the broader context there have been dramatic changes in the recent past to Aboriginal Torres Strait Islander society with loss of land, culture, language and lore. This led to changes in many traditions and values of communities.

3.2.3. Legal frameworks have changed. Aberrant behaviours, such as child sexual abuse would historically have been addressed by the Aboriginal Torres Strait Islander Peoples themselves with punishment in the form of 'Traditional Payback' (personal communication, Yugenbeh Elders, Logan Beaudesert and Gold Coast Aboriginal Torres Strait Islander Elders Group). Of course officially these laws are no longer practised. Australia's legal system now is similar to the British legal system.

3.2.4. It is tragic that abusive behaviours are ongoing, despite significant input from government and non government agencies. Despite numerous initiatives abusive behaviours have to remain a focus for statutory and non statutory agencies. We highlight three areas where we feel parallels could be drawn: an informed input may lead to changes in behaviour. The theories we draw on are: locus of control, motivational interviewing and building positive social norms. We feel these theories may assist the thinking how to reduce abuse of children.

3.2.4.1. An example for change in locus of control in recent history is where a community took ownership for the tragedy of repeated youth suicide and made a significant change. In the mid 1990's the Yarrabah Indigenous Community in North Queensland saw a suicide epidemic. 
Significant efforts were made by state and national specialist teams from Australia and some other countries (eg. Canada). These efforts did not lead to the desired suicide reduction. It was only when the Yarrabah people took the initiative into their own hands that the suicide rates dropped. Another example from the recent past is the life of Edward Mabo [19]. He was known for his initiative. He had an internal locus of control. Edward Mabo made a great difference to the lives of Australian indigenous people especially through political lobbying. Also the initiative of Aboriginal Torres Strait Islander Women in "Deeds of Grant in Trust" (DOGIT) is associated with internal locus of control. They aim to keep alcohol prohibited in remote and rural communities. Furthermore men's groups are a good example for fathers getting together and discussing aspects of mental health and welfare. Such groups have an enormous potential. Communities that take education about child management in their own hands may well contribute to reducing child abuse.

3.2.4.2. Motivational interviewing is a skill used by professionals to help individuals with e.g. addiction. It helps start thinking about changing behaviours. Maybe perpetrators from a motivational stages point of view [20] are in the stage of pre-contemplation. We hope that perpetrators with unacceptable behaviours will be able to move from the stage of pre-contemplation to contemplation and then hopefully to the stage of action and reform their behaviours. This would then be followed with maintenance of the acceptable behaviours.

3.2.4.3. At a societal level social norms can be positively influenced. Kelman's [21] building positive social norms postulates that firstly rules and regulation will have to be complied with, secondly individuals will then identify with rules and norms and thirdly individuals will internalise behaviours and concepts. This suggests that legal frameworks will eventually lead to changed behaviours but also changes in attitude by selected members in a group. Some individual may then influence their peers and so on.

\section{Potential of IT}

4.1. The world has seen rapid progress in internet technologies and world-wide communications [22]. It is already affecting the pace of societal progress; there are numerous examples of the power of modern technology. The common theme of changes triggered with the assistance of IT is that people without voice collaborate by IT networking and then exert influence as a group firstly in cyberspace and then the real world. Part of the power to trigger change may lie in its immediacy and easy access. IT already is widely available in many parts of the world; journalists and media assist in spreading the word.

4.2. One of the most recent and publicised examples was the Arabian spring in Egypt which allegedly started because of the grief of one street vendor with police and corruption. It is reported that then individuals from not-so-privileged backgrounds started a process, fanned by Facebook, which then supposedly lead to the revolution in Egypt. Facebook apparently assisted in spread of news and getting individuals rapidly together. As of 2012 there were almost 1 billion members of Facebook worldwide, with boundless opportunities for networking. Modern technology assists across the world.

4.3 Many initiatives for Aboriginal Torres Strait Islander peer group discussion already exist even without the use of IT services. For example, on Palm Island - an indigenous region in North Queensland - a women's group "Malanbarra Midja" is a forum where attendees may find peers, find support and may come to a position to effect societal change. We suggest that once telephone and internet services are rolled out Australia-wide, including remote areas that traditionally have been difficult to access, maximised access is likely to contribute to progress in many domains, making such groups as "Malanbarra Midja" more easily accessible. Possibly this could contribute to a reduction of child abuse.

\section{Barriers and limitations and how to overcome barriers and limitations}

There are a number of limitations when it comes to closing the Gap. This is at IT infrastructure, a wider community and political level, and individual level.

5.1 Limitations with respect to access to IT technologies are likely to be the geography, purchase and running costs. Despite Australia's strong drive to roll out nationwide broadband it remains to be seen whether the remotest parts of all States and Territories will be connected or not. It is difficult to imagine that indeed all of geographic Australia will one day have broadband access. It may not make economical sense to roll out broadband in desert areas where no one lives, and where very rarely only very few people are transiting? However remote parts of Australia where indigenous people live certainly could get well connected. So far IT access is limited to the most important metropolitan and regional areas, as can be seen on the Telstra - Australia's main telecommunication company - coverage maps [23]. Satellite phone access is limited by prohibitive costs. We are uncertain how realistic plans are that talk about balloons distributed in the stratosphere around the world making wifi accessible "everywhere".

5.2 Even if hardware, access and IT connections were in place, there are likely significant other hurdles to consider. We feel that we can learn from hurdles that researchers have identified when looking at communities. We refer to work by Marmot [24] and Kunitz [25].

Michael Marmot highlights the importance of ownership, respect from others, commitment to sufficient time frames, and accept the connectedness to past and country for Aboriginal Torres Strait Islanders. Marmot's ideal is 'provide the answers to key questions relating to health that might enable indigenous Australians to live the lives that they will choose to live'. Marmot differentiates between essential principles and desirable principles. The five essential principles are: address priorities how the community determines them, those who are not part of the 
community to interact with the community in mutually respectful partnership framework, provide sufficient resources to facilitate the process and assure appropriate longevity of the initiative, have appropriate flexibility to reach the goals in time, and respecting the community's past and present. The 5 desirable principles are: recognising the diverse people, accept there may be extensions to set time frames, accept there will be changes to indigenous leadership, support community ownership, and facilitate partnership.

Steven Kunitz referred to Anglo-settler societies in Australia, New Zealand, Canada and United States of America. He highlighted that past colonial history and policies are important in influencing and explaining differences in world-wide metrics like mortality rates, longevity and infant mortality, and therewith gave a paradigm for the health gap between indigenous and majority societies. He discussed six cultural continuity markers that are important to the independent control of indigenous societies: lands right, indigenous community control over education, health, police, cultural facilities, child welfare services and elected band councils with more than $50 \%$ women.

5.3.1. Other limitations are relevant to the child as an individual. We look at learning, access and cognitive development. There may be lack of exposure to novel opportunities. Usually children would have access to news in education setting. However in remote communities school attendance may be poor. Illiteracy may reduce the potential to make use of written information. Children with communication disorders will have difficulties expressing themselves and/or making use of heard language. Notwithstanding once children and young people have consistent access to internet, learning and training could be maximised with tutorials on for example "youtube". Access to IT may well be an important contributor to education and therewith changes in behaviour and attitudes. A further incentive to welcome internet may be access to the digital economy. Social networking, blogging may be of interest to the broader community too. Such helpful options are in competition with online gaming, gambling etc.

5.3.2. There may be risks associated with cyberspace opportunities that could be a limitation to children connecting on the internet. We refer to an example from the UK. In 2012 IT access facilitated public crime: smart phones assisted getting individuals together to start an, albeit brief, epidemic of street vandalism. It is with a mind-blowing speed that people got together. Authorities in the UK initially could not keep up with events and were not prompt enough to organise an appropriate response fast enough. Later perpetrators could be identified as calls, text messages and communications are logged. Eventually perpetrators may be identified and prosecuted. We suppose that numerous other examples can be thought of where bloggers organised people to come together and triggered change. An example where children are lead to activity and "mass gatherings" and queuing sometimes overnight without ulterior motive and not with criminal intent, are the queues when there is release of new children's book ( remember Harry Potter) or a new x-box or similar device. We are not going to expand on the risks to children from adults with unacceptable motives in for example chat rooms. This is being addressed elsewhere. Appropriate supervision may be a helpful way addressing these risks.

5.3.3 When talking about children we need to keep child development in mind. It depends on the developmental and chronological age as to what is possible for children to implement changes. Piaget [26] developed neuro-cognitive developmental models which show that until in their later teens, for neurocognitive reasons children are unlikely to speak up for themselves. Younger children will have typically an external locus of control; this is influenced by culture, ethnicity and also cognitive moral development [27]. Legislative bodies are aware of this and may provide for children to have advocates, for example in legal or police matters. Stages of development associated with questioning values around the time of puberty likely lead to intrinsic questioning of values that have been passed down from the older generations [28]. Values are influenced by the environment i.e. family, peers and friends and school education. Chronological age and developmental stage need to be borne in mind when expectations are expressed in how far "children" can stand up for themselves. The older a child is and the better developed the earlier a child can take initiative.

For children who suffered trauma a limitation, in addition to development as aforementioned, may be the response to trauma. Whilst some victims may be resilient to trauma, others may be very vulnerable and develop more impairment compared to those who are resilient. To a victim trauma can have impact in many domains. Not infrequently a traumatised child victim may suffer from the sequelea of trauma. Post traumatic stress disorder may present with intrusive memories, nightmares and avoidance of aspects in life that remind of trauma as well as hypervigilance. Beyond PTSD after trauma a child may present as helpless. This may appear to an outstanding observer as inertia. Hence the psychological effects of trauma can be important limitations to the victim asserting him/herself. This can be addressed. According to P. Crittenden [29] just as the after-effects of trauma can be multi-fold any response must also be multi-level and comprehensive. This is not to say that an individual with either little or much resilience will not recover by him/herself. However the right environment is supremely important. Stability, consistency and predictability should be promoted. Relationships and especially the dyadic relationships between children and the key individuals in their lives should be maximised. With stability in life, permanency and consistency in relationships feelings of safety may resume, trust to communicate develops, this can lead to communication of deep and painful memories and real progress to a "normal life" may start once the victim has come to terms with injustices and has started the process of: leaving behind the not forgiving. Anger and hate can perpetuate subjective distress similar to a cancer. In 
this sense healing on the inside for the victim should be seen as separate from the internal world of the perpetrator. (A perpetrator may have to go a different path to recovery. With reflective abilities perpetrators may start forgiving themselves [30].) Hence assisting and enhancement of relationships between individuals and institutions involved with young people is a good start. Even in the face of trauma and developmental issues we hope that eventually locus of control can change leading to a positive and constructive response.

5.3.4. An important additional limitation is drug and alcohol use. These substances have sedative effects, can also impair thinking processes, can distort views of reality, and reduce assertiveness with volition. Individuals must be advised against illicit drug and alcohol use [31], and other physical, developmental and mental health issues must be addressed.

5.3.5. Limitations to the validity of this article as a whole are: we present previously ideas in a new combination. It is an empirical article. This is not evidence based research in the sense of evidence based medicine. However the hypothesis presented may compensate in the short term until research studies have been published that can be generalised to all Australia's groups where locus of control was put in context of IT access and child protection and development. Indeed an answer may be opening this topic to formal research. For example it would be desirable to review the prevalence of child protection referral year per year after remote and rural areas IT access has improved. Furthermore and notwithstanding the power of randomised trials and meta-analysis etc we refer to a report by Anthropologist John von Sturmer [32]. This case study shows how internal locus of control can lead to new behaviours that can be observed by all those around: "Males were not involved with their children, my partner was not involved in raising any of our children, now you see fathers walking their children to school and supporting their partners when they have difficult times with the children. The next biggest change has been that everyone is starting to see what happens in the community as their responsibility. Other changes have been that people are realising that everyone has rights, especially children."

\section{Conclusions}

Australia is a nation with a very long history going back 10 thousands of years. Present day society continues as multicultural and multiethnic. Although Aboriginal Torres Strait Islanders can be at an advantage in many domains they often are at a disadvantage called "the gap". We showed how the gap applies also to provision of internet access, especially in remote areas. We discussed locus of control and internal locus of control in its wider context. We showed that internet, managed wisely and appropriately supervised, can be beneficial and inspire people into action. We gave examples where we feel there may be opportunities for abusers to change their behaviours. We contribute to the discussion about the use and misuse, the advantages and disadvantages of technologies. We present an optimistic view. We acknowledged limitations and offered exit strategies. We gave examples as to how those who historically did not have a voice have now a forum through IT. This may then have beneficial effects on rural as well as metropolitan areas in Australia. Ideally benefits would accumulate: reduced costs in terms of impairment and handicap to individuals (and also financial costs to the state), access to education, resisting alcohol and drugs, literacy, resilience to trauma, good physical and mental health, direct peer and family support, safe and nurturing role models, secure attachment, internal locus of control. It is about moving forward together for the protection of the youngest members of society.

\section{Acknowledgements}

Martin and Adrian both have a teaching commitment with Griffith University Australia. Martin is a Psychiatrist, originally from Europe, specialising in the mental health of children. Adrian has a nursing background, he grew up in Queensland. Both Adrian and Martin are committed to the development of science and the passing on of knowledge to future generations of health professionals for the benefit of patients. Both Adrian and Martin have a very keen interest in prevention, especially prevention of harm to children. Both acknowledge the Traditional Owners on whose lands we live and work on. We both pay our respects to Elders past and present.

\section{Conflict of Interests}

The authors have no conflicts of interests to declare.

\section{REFERENCES}

[1] Australian Institute of Health and Welfare. [Homepage on the internet].cited 2013 June 14].Available from:

http://www.aihw.gov.au/publications/

[2] Darwin C. The Origin of Species by Means of Natural Selection: Or, The Preservation of Favoured Races in the Struggle for Life. John Murray ; 1872.

[3] Roeg, N., Bond E, Marshall JV. Walkabout. 20th Century Fox; 1971 .

[4] DATSIMA [Homepage on the internet]. Closing the Gap Targets. Queensland Government, Department of Aboriginal and Torres Straight Island and Multicultural Affairs, Aboriginal and Torres Straight Islander Affairs; 2007/2008 [cited 2013 February 2].Availablefrom: http://www.datsima. qld.gov.au/resources/atsis/government/programs-initiatives/c losing-gap/2008-09-annual-report/ctg-0809-intro-targets-key ind.pdf 
[5] Thornton N. Samson and Delilah. Screen Australia, New South Wales Film and Television Office, Scarlett Pictures Pty Ltd, Warwick Thornton \& CAAMA Productions Pty Ltd ; 2009.

[6] Graham S, Guy RJ, Donovan B. Epidemiology of chlamydia and gonorrhoea among Indigenous and non-Indigenous Australians, 2000-2009. Medical Journal Australia; 2012.

[7] Bygott J, Robson J M. The rarity of Trichomonas vaginalis in urban Australia. Sexual Transmitted Infections; 2013.

[8] Hunter E. Indicators of psychoses or psychoses as indicators: the relationship between Indigenous social disadvantage and serious mental illness. Australasian Psychiatry; 2013.

[9] Heart Foundation. [Homepage on the internet].closing the gap [cited 2013February2].Availablefrom: http://www.heartfoun dation.org.au/information-for-professionals/aboriginal-health /Pages/default.aspx

[10] Bradford - Canon W. The Wisdom of the body. Norton Library; 1932.

[11] Philip G, Ney MD . Transgenerational child abuse. Child Psychiatry \& Human Development ; 1988.

[12] Battle E, Rotter JB. Children's feelings of personal control as related to social class and ethnic group. Journal of Personality. Vol 31(4): 482-490. 1963

[13] Piaget J. The development of thought: Equilibration of cognitive structures. Trans A. Rosin. Oxford, England: Viking. 1977.

[14] Hiroto DS, Seligman ME. Generality of learned helplessness in Journal of Personality and Social Psychology, Vol 31(2), Feb 1975, 311-327.1975.

[15] Streeck-Fischer A, van der Kolk B. Down will come baby, cradle and all: Diagnostic and therapeutic implications of chronic trauma on child development. Aust NZ J Psychiatry. 34(6):903-918. 2000

[16] Schore, A. Affect regulation and the origin of the self: the neurobiology of emotional development. Hillsdale, NJ: Lawrence Erlbaum Associates. 1994.

[17] Perini S, Titov N, Andrews, G. Clinician-assisted Internet-based treatment is effective for depression: Randomized controlled trial. Vol. 43, No. 6 , Pages 571-578. 2009.

[18] NBNCO. National Broadband Network. [Homepage on the internet]. [cited 2013 February 2]. Available from: Http://www.nbnco.com.au/

[19] Mabo, E. Indigenous Australian case study: Torres Strait Islands, Mer (Murray Island) and Eddie 'Koiki' Mabo. Told by his daughter Gail Mabo. [cited2013February2].Availablef rom:

http://hsc.csu.edu.au/ab_studies/rights/global/social_justice global/sjwelcome.response.front.htm\#Indigenous\% $\%$ 20Austral ian\%20Case \%20Study

[20] Miller WR, Rollnick S. Motivational interviewing, Preparing people for change. The Guilford Press: New York, NY. 1991.

[21] Kelman HC. Three processes of social influence. Public Opinion Quarterly. 1961

[22] Castells M. The Information Age: Economy, Society, and
Culture. Vol. 2, The Power of Identity. Oxford: Blackwell. 1997.

[23] Telstra. telstra broadband coverage. [cited 2013 February 2]. Available from:http:/www.telstra.com.au/mobile-phones/co verage-networks/our-coverage/mobile-broadband/

[24] Marmot M. Social determinants and the health of Indigenous Australians. Medical Journal Australia; 194: 512-513. 2011.

[25] Kunitz SJ. Disease and social diversity: the European impact on the health of non-Europeans. New York: Oxford University Press.1994.

[26] Piaget J. The child 's conception of physical causality . Transaction publishers: New Jersey. 1930.

[27] Kohlberg L. The philosophy of moral development : moral stages and the idea of justice. Chicago.1981

[28] Blakemore S, Choudhury S. Development of the adolescent brain:implications for executive function and social cognition .Journal of Child Psychology and Psychiatry. 47:3 : 296-312. 2006.

[29] Crittenden PM. Treatment of anxious attachment in infancy and early childhood. Developmental Psychopathology. 4: 575-602. 1992.

[30] Schechter DS, Coots T, Zeanah C, Davies M, Coates SW, Trabka KA, Marshall RD, Liebowitz MR, Myers MM. Maternal mental representations of the child in an inner-city clinical sample: Violence-related posttraumatic stress and reflective functioning. Attachment \& Human Development. 7, 3, pp 313-331.2005.

[31] Department of Aboriginal and Torres Strait Islander multicultural affairs. [cited 2013 February 2]. Alcohol Management Plan. Available from : http://www.datsima.qld. gov.au/atsis/government/programs-and-initiatives/review-ofalcohol-management-plans-2012

[32] Von Sturmer J, Le Marseny S. Living under the Family Responsibilities Commission: Experience and testimony, 'speaking straight, speaking from the heart' summary report, p. 15. 2013. 